\title{
Health behaviors and their correlates among participants in the Continuing to Confront COPD International Patient Survey
}

This article was published in the following Dove Press journal:

International Journal of COPD

27 April 2016

Number of times this article has been viewed

\author{
Hana Müllerová \\ Sarah H Landis' \\ Zaurbek Aisanov ${ }^{2}$ \\ Kourtney J Davis ${ }^{3}$ \\ Masakazu Ichinose 4 \\ David M Mannino ${ }^{5}$ \\ Joe Maskell' \\ Ana M Menezes ${ }^{6}$ \\ Thys van der Molen ${ }^{7}$ \\ Yeon-Mok $\mathrm{Oh}^{8}$ \\ Maggie Tabberer 9 \\ MeiLan K Han ${ }^{10}$ \\ 'GSK, Worldwide Epidemiology, \\ Uxbridge, UK; ${ }^{2}$ Pulmonology Research \\ Institute, Moscow, Russia; ${ }^{3}$ GSK, \\ Worldwide Epidemiology, Upper \\ Providence, PA, USA; ${ }^{4}$ Tohoku \\ University Graduate School of \\ Medicine, Sendai, Japan; ${ }^{5}$ University \\ of Kentucky College of Public \\ Health, Lexington, KY, USA; ${ }^{6}$ Federal \\ University of Pelotas, Pelotas, Brazil; \\ ${ }^{7}$ University of Groningen, University \\ Medical Center Groningen, Groningen, \\ the Netherlands; ${ }^{8}$ University of Ulsan \\ College of Medicine, Asan Medical \\ Center, Seoul, South Korea; ' $\mathrm{GSK}$, \\ Value Evidence Outcomes, Uxbridge, \\ UK; ${ }^{10}$ Division of Pulmonary and \\ Critical Care, University of Michigan, \\ Ann Arbor, MI, USA
}

Correspondence: Hana Müllerová Real World Evidence \& Epidemiology, GSK, Stockley Park West, I-3 Ironbridge Road, Uxbridge, Middlesex,

UBI I IBT, UK

Email hana.x.muellerova@gsk.com
Background and aims: We used data from the Continuing to Confront COPD International Patient Survey to test the hypothesis that patients with COPD who report less engagement with their disease management are also more likely to report greater impact of the disease.

Methods: This was a population-based, cross-sectional survey of 4,343 subjects aged $\geq 40$ years from 12 countries, fulfilling a case definition of COPD based on self-reported physician diagnosis or symptomatology. The impact of COPD was measured with COPD Assessment Test, modified Medical Research Council Dyspnea Scale, and hospital admissions and emergency department visits for COPD in the prior year. The 13-item Patient Activation Measure (PAM-13) instrument and the 8-item Morisky Medication Adherence Scale (MMAS-8) were used to measure patient disease engagement and medication adherence, respectively.

Results: Twenty-eight percent of subjects reported being either disengaged or struggling with their disease (low engagement: PAM-13 levels 1 and 2), and 35\% reported poor adherence (MMAS-8 <6). In univariate analyses, lower PAM-13 and MMAS-8 scores were significantly associated with poorer COPD-specific health status, greater breathlessness and lower BMI (PAM-13 only), less satisfaction with their doctor's management of COPD, and more emergency department visits. In multivariate regression models, poor satisfaction with their doctor's management of COPD was significantly associated with both low PAM-13 and MMAS-8 scores; low PAM-13 scores were additionally independently associated with higher COPD Assessment Test and modified Medical Research Council scores and low BMI (underweight).

Conclusion: Poor patient engagement and medication adherence are frequent and associated with worse COPD-specific health status, higher health care utilization, and lower satisfaction with health care providers. More research will be needed to better understand what factors can be modified to improve medication adherence and patient engagement.

Keywords: chronic obstructive pulmonary disease, patient survey, patient engagement, adherence

\section{Introduction}

When managing chronic diseases, understanding the patients' perspective in terms of expectations, concerns, and beliefs about their disease could result in better patientphysician communications and consequently improve management of the disease. ${ }^{1}$ Personality traits and health care beliefs are likely to affect patient perceptions of care and could in turn influence the understanding between patients and physicians. These types of patient traits have been defined as "patient's engagement" and can be quantified using survey instruments, such as the Patient Activation Measure (PAM). ${ }^{2}$ 
Studies in patients with chronic conditions have shown that greater engagement (higher PAM score) is associated with an improvement in patient health-related behaviors. ${ }^{3,4}$ These data suggest that patients who are better engaged with their disease by actively participating in their own care are more likely to have better health outcomes. For example, programs that promote self-management or patient-centered chronic care initiatives in COPD have been shown to reduce health care resource utilization, including COPD-related hospital admissions..$^{5,6}$

Patient engagement with their disease can also be reflected in reported or measured adherence to prescribed medication. Good adherence to prescribed COPD medication has been associated with reduced all-cause mortality and hospital admissions due to COPD exacerbations. ${ }^{7,8}$ However, adherence among COPD patients is often poor. ${ }^{9-13}$

We hypothesized that COPD patients who report lower levels of engagement or medication adherence would report a greater burden of COPD in terms of humanistic impact (health status), symptom control, and health care utilization than patients who report greater engagement or adherence. Further, we aimed to identify factors associated with patients' level of engagement with COPD and adherence with COPD treatment.

\section{Methods}

\section{Study design}

The study objectives that were explored in the Continuing to Confront COPD International Patient Survey, have been described previously; ${ }^{14}$ a detailed description of the survey sampling methods can be found in the online appendix. This was a population-based survey of 4,343 adults who fulfilled a case definition of COPD defined as adults aged 40 years and older who reported 1) a physician diagnosis of COPD/ emphysema, 2) a physician diagnosis of chronic bronchitis, or 3 ) met a symptom-based definition of chronic bronchitis and were either taking respiratory medication for their condition or had chronic cough with phlegm most days. A history of smoking was not required as part of the case definition for this study. The survey was conducted in 12 countries; Brazil, France, Germany, Italy, Japan, Mexico, the Netherlands, Russia, South Korea, Spain, the UK, and the US.

Participation in the survey was entirely voluntary and confidential, and all subject data were anonymous. The survey protocol and consent procedure were reviewed by the Abt SRBI Institutional Review Board (registered with the Office for Protection from Research Risks, Health and Human Services) and granted an Institutional Review Board exemption as the criteria for exemption under 45 CFR 46.101(b) (2) of the United States Code of Federal Regulations were met. In countries where face-to-face interviews were conducted in the respondents' home, modest incentives may have been offered, the value of which was less than US\$6 (eg, mobile phone top-up card, tea, chocolates). In Japan, the members of the online panel were compensated by the panel provider directly, typically $\$ 11$. No other incentives were provided.

Patients completed a structured questionnaire about their COPD disease severity, respiratory symptoms, treatment for COPD, health care resource use for exacerbations of COPD in the prior 12 months (number of emergency department visits and number of hospitalizations), smoking history and toxic exposures, and overall satisfaction with their doctor's management and treatment of COPD. Symptom severity was assessed using the modified Medical Research Council (mMRC) Dyspnea Scale, ${ }^{15}$ and health status with the COPD Assessment Test (CAT). ${ }^{16,17}$ The primary hypotheses were addressed using the PAM-13, ${ }^{2}$ and Morisky Medication Adherence Scale (MMAS)-8. ${ }^{18}$ Validated translations of the patient reported outcome instruments were used and permission for their use was obtained.

\section{Patient Activation Measure-13}

The PAM-13 was used to measure patients' self-confidence in their role in managing their disease and assertiveness in dealing with the health care systems. ${ }^{2}$ This scale evaluates patients' responsibility, attitude, confidence, knowledge, and understanding of their disease.

PAM-13 yields a scaled score ranging from 0 to 100 that assigns a patient to one of four levels:

- Level 1 (PAM-13 score of 47.0 or lower): disengaged and overwhelmed

- Level 2 (PAM-13 score of 47.1 to 55.1): becoming aware but still struggling

- Level 3 (PAM-13 score of 55.2 to 67.0): taking action

- Level 4 (PAM-13 score of 67.1 or above): maintaining behaviors and pushing further.

\section{Morisky Medication Adherence Scale-8}

The MMAS-8 is an 8-item, self-reported measure of adherence in recent users of medication (recall period is 14 days $).{ }^{18}$

Each question is given either a dichotomous or Likert scale-type response resulting in an overall score which ranges from 1 to 8 , with higher scores indicating greater adherence. Adherence is classified as low (score 1-5), 
medium (score 6-7), or high (score 8) according to the overall score.

\section{Statistical analyses}

Analyses related to patient engagement were conducted in 4,339 respondents. Analysis of patient adherence was limited to 3,135 respondents who reported taking medication in the past 14 days and thus were eligible to complete MMAS-8.

All analyses were weighted using a summary survey weight variable to address factors of age and sex sampling specific to each country and also to adjust for the proportion of each country's contribution to the study population. Therefore, all percentages presented in the paper are weighted percentages. Differences in demographic, clinical, and behavioral traits across levels of PAM-13 and MMAS-8 were tested using weighted chi-square test for categorical variables and proportional odds model for continuous variables. Weighted logistic regression multivariate models (PROC SURVEYLOGISTICS) were used to determine factors associated with (a) poor adherence (MMAS-8 score $<6$ ) and (b) poor patient engagement
(PAM-13 levels 1 and 2) using characteristics showing a significant univariate relationship with each respective outcome and country. All analyses were conducted in SAS v9.3.

\section{Results}

In the population of 4,343 respondents who fulfilled the survey definition of COPD, the mean age was 60.7 years (standard deviation 12.1 years), 48\% were male, and 64\% were current or former smokers. ${ }^{14}$ Distribution of traits in the subsample not completing the MMAS- 8 was mostly identical or differed nonsignificantly (data not shown).

\section{Patient engagement with COPD management and its determinants}

The majority of respondents with valid PAM-13 scores $(\mathrm{N}=4,339)$ indicated high PAM-13 scores with 51\% scoring at the highest Level 4 and 22\% at Level 3 (Table 1). However, $15 \%$ of respondents were disengaged and overwhelmed by their disease (Level 1) and 13\% described themselves as struggling with their disease (Level 2). Weighted mean value for the PAM-13 score was 66.75 (standard deviation 17.90).

Table I Characteristics of respondents with COPD according to activation level as measured with Patient Activation Measure (PAM)-I3

\begin{tabular}{|c|c|c|c|c|c|}
\hline \multirow[t]{2}{*}{$\overline{\text { Characteristic }^{\wedge}}$} & \multicolumn{4}{|l|}{ PAM-I 3 level } & \multirow{2}{*}{$\begin{array}{l}\text { P-value across } \\
\text { PAM-I3 } \\
\text { levels }^{*}\end{array}$} \\
\hline & $\begin{array}{l}\text { Level I: disengaged } \\
\text { and overwhelmed } \\
N=639(15 \%)\end{array}$ & $\begin{array}{l}\text { Level 2: } \\
\text { becoming aware } \\
N=56 \text { I (13\%) }\end{array}$ & $\begin{array}{l}\text { Level 3: } \\
\text { taking action } \\
\mathrm{N}=946(22 \%)\end{array}$ & $\begin{array}{l}\text { Level 4: maintaining } \\
\text { behaviors } \\
N=2,193(51 \%)\end{array}$ & \\
\hline Females & 49.4 & 51.2 & 48.6 & 54.6 & 0.010 \\
\hline Males & 50.6 & 48.8 & 51.4 & 45.4 & \\
\hline \multicolumn{6}{|l|}{ Age (years) } \\
\hline $40-59$ & 45.2 & 51.6 & 49.5 & 48.1 & 0.653 \\
\hline $60+$ & 54.8 & 48.4 & 50.5 & 51.9 & \\
\hline \multicolumn{6}{|l|}{ Smoking status } \\
\hline Never smoker & 38.4 & 42.5 & 35.5 & 33.3 & 0.030 \\
\hline Former smoker & 31.4 & 35.0 & 36.9 & 38.8 & \\
\hline Current smoker & 30.2 & 22.6 & 27.6 & 27.9 & \\
\hline BMI $\left(\mathrm{kg} / \mathrm{m}^{2}\right)$, mean $(\mathrm{SD})$ & $26.2(5.9)$ & $26.0(6.2)$ & $26.4(6.0)$ & $27.6(6.6)$ & $<0.000$ I \\
\hline CAT score, weighted mean (SD) & $23.7(8.8)$ & $21.3(9.7)$ & $20.3(8.9)$ & $21.3(9.3)$ & $<0.001$ \\
\hline \multicolumn{6}{|l|}{ CAT impact category } \\
\hline Low impact $(1-10)$ & 9.1 & 16.6 & 16.7 & 14.7 & $<0.0001$ \\
\hline Medium impact (II-20) & 23.5 & 27.8 & 31.9 & 29.9 & \\
\hline High impact (2I-30) & 43.7 & 35.4 & 39.1 & 37.6 & \\
\hline Very high impact $(3 \mid-40)$ & 23.7 & 20.1 & 12.3 & 17.8 & \\
\hline \multicolumn{6}{|l|}{ mMRC Dyspnea score } \\
\hline $0-1$ & 44.0 & 53.9 & 59.4 & 52.3 & $<0.01$ \\
\hline $2-4$ & 56.0 & 46.1 & 40.6 & 47.7 & \\
\hline \multicolumn{6}{|l|}{$\begin{array}{l}\text { Self-reported history of past } \\
\text { spirometry }\end{array}$} \\
\hline Yes & 70.2 & 71.4 & 74.8 & 81.4 & $<0.0001$ \\
\hline No & 29.8 & 28.6 & 25.2 & 18.6 & \\
\hline
\end{tabular}


Table I (Continued)

\begin{tabular}{|c|c|c|c|c|c|}
\hline \multirow[t]{2}{*}{ Characteristic $^{\wedge}$} & \multicolumn{4}{|l|}{ PAM-I3 level } & \multirow{2}{*}{$\begin{array}{l}\text { P-value across } \\
\text { PAM-I3 } \\
\text { levels }^{*}\end{array}$} \\
\hline & $\begin{array}{l}\text { Level I: disengaged } \\
\text { and overwhelmed } \\
\mathrm{N}=639(15 \%)\end{array}$ & $\begin{array}{l}\text { Level 2: } \\
\text { becoming aware } \\
\mathrm{N}=56 \text { I (13\%) }\end{array}$ & $\begin{array}{l}\text { Level 3: } \\
\text { taking action } \\
\mathrm{N}=946(22 \%)\end{array}$ & $\begin{array}{l}\text { Level 4: maintaining } \\
\text { behaviors } \\
\mathrm{N}=2,193(51 \%)\end{array}$ & \\
\hline \multicolumn{6}{|l|}{ Number of comorbidities } \\
\hline $0-1$ & 66.9 & 73.9 & 72.0 & 62.2 & $<0.0001$ \\
\hline$\geq 2$ & 33.1 & 26.1 & 28.0 & 37.8 & \\
\hline \multicolumn{6}{|l|}{ Number of COPD medications } \\
\hline $0-1$ & 65.8 & 61.6 & 59.9 & 58.3 & 0.0007 \\
\hline$\geq 2$ & 34.2 & 38.4 & 40.1 & 41.7 & \\
\hline \multicolumn{6}{|l|}{ Emergency department visits } \\
\hline \multicolumn{6}{|l|}{ for COPD in past 12 months } \\
\hline None & 76.9 & 78.3 & 80.4 & 73.2 & 0.007 \\
\hline$\geq 1$ & 23.1 & 21.7 & 19.6 & 26.8 & \\
\hline \multicolumn{6}{|l|}{ Hospital admissions for COPD in past } \\
\hline \multicolumn{6}{|l|}{12 months } \\
\hline None & 80.2 & 85.9 & 87.0 & 84.7 & 0.053 \\
\hline$\geq 1$ & 19.8 & 14.1 & 13.0 & 15.3 & \\
\hline \multicolumn{6}{|l|}{ Education level } \\
\hline Elementary school & 55.5 & 55.0 & 55.6 & 58.0 & 0.143 \\
\hline $\begin{array}{l}\text { Higher education/technical college } \\
\text { or further education }\end{array}$ & 44.5 & 45.0 & 44.4 & 42.0 & \\
\hline \multicolumn{6}{|l|}{$\begin{array}{l}\text { Satisfaction with doctors management } \\
\text { of COPD }\end{array}$} \\
\hline Very satisfied & 25.4 & 33.6 & 41.3 & 66.3 & $<0.000 \mathrm{I}^{\#}$ \\
\hline \multicolumn{6}{|l|}{ Involved in decisions about treatment } \\
\hline Strongly agree & 19.8 & 25.6 & 34.9 & 65.9 & $<0.000 \mathrm{I}^{\#}$ \\
\hline \multicolumn{6}{|l|}{ Treatment plan or goals set up } \\
\hline Strongly agree & 17.4 & 18.5 & 26.8 & 56.6 & $<0.000 \mathrm{I}^{\#}$ \\
\hline
\end{tabular}

Notes: ^Presented as column weighted percent unless otherwise stated; ${ }^{*}$ tested across PAM-I 3 levels using weighted chi-square test, proportional odds model or weighted ANOVA; ${ }^{P}$-value derived across all response categories.

Abbreviations: SD, standard deviation; CAT, COPD Assessment Test; mMRC, modified Medical Research Council.

Univariate analysis showed that patients with low PAM-13 scores were more often male, had lower BMI, reported worse COPD-specific health status (higher CAT score), worse breathlessness on exertion ( $\mathrm{mMRC} \geq 2$ ), were less likely to have undergone a past spirometry test, and reported taking fewer COPD medications (Table 1). Patients with low PAM-13 scores were also more likely to report that they were "somewhat or very dissatisfied" with how their physician manages their disease $(P<0.001)$ (Figure 1; Table 1) and were less likely to report being involved in treatment decisions/having a disease management plan $(P<0.001)$ (Table 1). In the multivariate model (using characteristics showing a significant univariate relationship with each respective outcome and including country), independent factors associated with low levels of engagement (PAM-13 Levels 1 and 2) included increased breathlessness (mMRC score $\geq 1)$, lower patient satisfaction with their doctor, low BMI ( $<18.5 \mathrm{~kg} / \mathrm{m}^{2}$; underweight), and poorer COPD-specific health status (higher CAT score) (Table 2).

\section{Patient reported adherence with COPD treatment and its determinants}

Patient adherence results were based on 3,135 (72\%) of respondents who reported taking medication in the past 14 days and had completed the MMAS- 8 . Low MMAS- 8 scores (poor adherence) were reported by $39 \%$ of patients, with $35 \%$ reporting medium and $27 \%$ high adherence (Table 3).

Patients with low MMAS-8 score were younger, more often females, reported poorer COPD-specific health status (higher CAT scores), were less likely to have undergone a past spirometry test, and were slightly more likely to have attended an emergency department in the previous 12 months (Table 3). Patients reporting low or medium adherence more often reported having $\geq 2$ comorbidities compared with those reporting high adherence. Poor adherers were also more likely to report that they were "somewhat or very dissatisfied" with how their doctors manage their COPD $(P<0.001)$ (Figure 2; Table 3) and were less likely to report being 

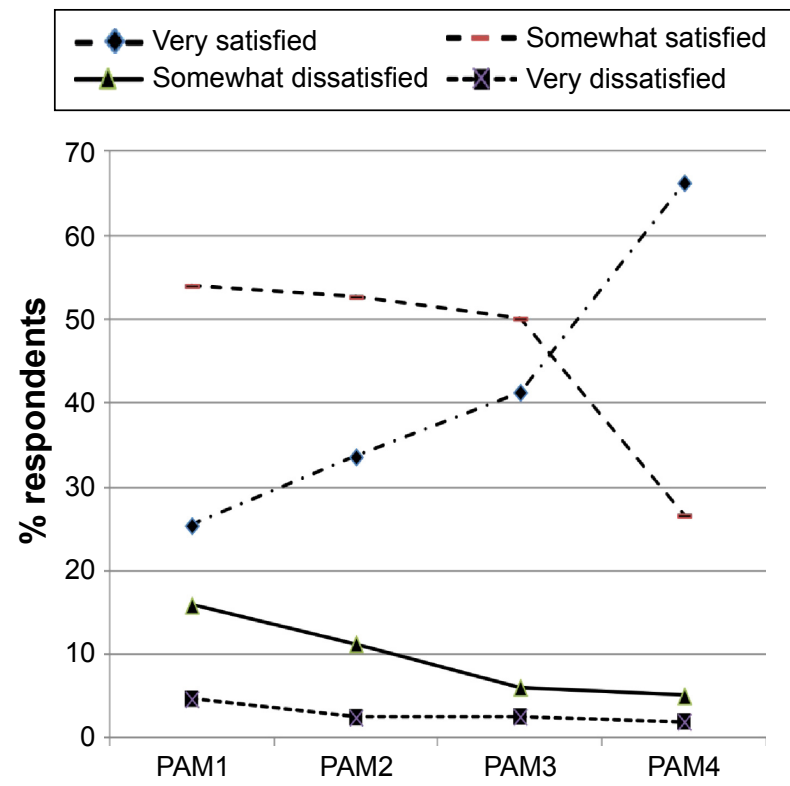

Figure I Satisfaction of patients with their treating physicians stratified by Patient Activation Measure (PAM)- 13 level.

Note: Respondent \% based on PAM level, that is, sum of proportions within each PAM level add up to $100 \%$.

involved in treatment decisions/having a disease management plan (both $P<0.001$ ) (Table 3). In the multivariate analysis, independent predictors of low treatment adherence included younger patients, current smokers, less satisfaction with their

Table 2 Factors associated with low disease activation among respondents with COPD (Patient Activation Measure Level I and 2)

\begin{tabular}{ll}
\hline Effect & $\begin{array}{l}\text { Odds ratio point } \\
\text { estimate (95\% Wald CL) }\end{array}$ \\
\hline $\begin{array}{l}\text { Age (years) } \\
40-59\end{array}$ & $\begin{array}{l}\text { I.II }(0.89-1.38) \\
\text { Age } \geq 60\end{array}$ \\
Sex & Ref \\
Female & $1.17(0.95-1.46)$ \\
Male & Ref \\
Smoking status & \\
Former smoker & $0.95(0.73-1.22)$ \\
Current & Ref \\
Never & $1.19(0.91-1.54)$ \\
BMI (kg/m $)$ & \\
$<18.5$ & $1.77(1.01-3.09)^{*}$ \\
I8.5-24.9 & Ref \\
$25-29.9$ & $1.16(0.91-1.47)$ \\
$>30$ & $0.98(0.74-1.30)$ \\
mMRC grade & \\
0 & Ref \\
I & $1.35(1.01-1.82)^{*}$ \\
2 & $1.72(1.25-2.36)^{*}$ \\
3 & $2.16(1.47-3.19)^{*}$ \\
4 & $1.87(1.07-3.27)^{*}$ \\
\hline &
\end{tabular}

Table 2 (Continued)

\begin{tabular}{ll}
\hline Effect & $\begin{array}{l}\text { Odds ratio point } \\
\text { estimate (95\% Wald CL) }\end{array}$ \\
\hline $\begin{array}{l}\text { Emergency department visits for } \\
\text { COPD, prior year }\end{array}$ & Ref \\
$\quad$ None & I.2I $(0.93-1.57)$ \\
$\geq 1$ & Ref \\
Satisfaction with doctors & $3.52(2.5-6.83)^{*}$ \\
$\quad$ Very satisfied & $2.12(1.69-2.67)^{*}$ \\
$\quad$ Somewhat satisfied & \\
Somewhat/very dissatisfied & I.05 $(1.02-1.08)^{*}$ \\
CAT score & \\
$\quad$ Per 2-unit increment & \\
Self-reported history of past & \\
spirometry & $0.83(0.65-1.05)$ \\
Yes & Ref \\
No & \\
Number of comorbidities & Ref \\
$0-1$ & $0.97(0.77-1.22)$ \\
$\geq 2$ & \\
Number of COPD medications & Ref \\
$0-1$ & $0.83(0.67-1.02)$ \\
$\geq 2$ &
\end{tabular}

Notes: The multivariate model was further adjusted for individual country. ${ }^{*}$ This variable remained statistically significant in the multivariate model $(95 \% \mathrm{CL}$ does not include I, $P<0.05$ ).

Abbreviations: CAT, COPD Assessment Test; CL, confidence limits; mMRC, modified Medical Research Council; Ref, reference stratum.

doctor's care, and $\geq 2$ comorbidities, but not COPD-specific health status or emergency department visits (Table 4).

\section{Discussion}

In our analysis of the Continuing to Confront COPD International Patient Survey, we report the first data on patient engagement in a large sample of respondents fulfilling the study definition of COPD. We observed that almost a third (28\%) of respondents struggled to understand their COPD and were not actively engaged in the management of their disease as indicated by low PAM-13 scores. Further, a large number of respondents (39\%) reported poor adherence with COPD medications as indicated by low MMAS- 8 scores. COPD patient disengagement and lack of adherence to COPD medications were associated with greater symptom burden and poorer satisfaction with health care providers.

In a survey of a representative general population sample of almost 18,000 adult residents of the US, $41 \%$ of respondents reported the highest level of engagement, ${ }^{19}$ comparable with our estimate of $50 \%$ of respondents reporting the highest level of engagement. However, in our survey, more than twice as many patients reported the lowest level of engagement ( $15 \%$ vs $7 \%$ ). Low engagement has also been reported to be highly prevalent among patients with other 
Table 3 Characteristics of respondents with COPD according to adherence levels as measured with the Morisky Medication Adherence Scale (MMAS)-8

\begin{tabular}{|c|c|c|c|c|}
\hline \multirow[t]{2}{*}{ Characteristic $^{\wedge}$} & \multicolumn{3}{|l|}{ MMAS-8 scale } & \multirow{2}{*}{$\begin{array}{l}P \text {-value across } \\
\text { MMAS-8 levels }\end{array}$} \\
\hline & $\begin{array}{l}\text { Low adherence } \\
(<6) \mathrm{N}=1,065\end{array}$ & $\begin{array}{l}\text { Medium adherence } \\
(6 \text { to }<8) \mathrm{N}=1,096\end{array}$ & $\begin{array}{l}\text { High adherence } \\
\text { (8) } N=974\end{array}$ & \\
\hline Females & 53.7 & 53.6 & 47.6 & 0.011 \\
\hline Males & 46.3 & 46.4 & 52.4 & \\
\hline \multicolumn{5}{|l|}{ Age (years) } \\
\hline $40-59$ & 56.0 & 41.5 & 34.9 & $<0.001$ \\
\hline $60+$ & 44.0 & 58.5 & 65.1 & \\
\hline \multicolumn{5}{|l|}{ Smoking status } \\
\hline Never smoker & 35.2 & 36.6 & 31.3 & 0.254 \\
\hline Former smoker & 34.6 & 43.9 & 45.3 & \\
\hline Current smoker & 30.2 & 19.8 & 23.5 & \\
\hline BMI $\left(\mathrm{kg} / \mathrm{m}^{2}\right)$, mean $(\mathrm{SD})$ & $27.2(6.7)$ & $27.1(6.9)$ & $26.7(5.6)$ & 0.258 \\
\hline CAT score, weighted mean (SD) & $23.6(9.2)$ & $23.2(8.9)$ & $21.4(8.6)$ & $<0.001$ \\
\hline \multicolumn{5}{|l|}{ CAT impact category } \\
\hline Low impact $(I-I 0)$ & 9.0 & 10.9 & 14.9 & $<0.001$ \\
\hline Medium impact (I I-20) & 24.0 & 25.5 & 28.8 & \\
\hline High impact $(2 \mid-30)$ & 43.5 & 41.4 & 38.9 & \\
\hline Very high impact $(3 \mid-40)$ & 23.6 & 22.3 & 17.3 & \\
\hline \multicolumn{5}{|l|}{ mMRC dyspnea score } \\
\hline $0-1$ & 48.1 & 48.1 & 46.7 & 0.569 \\
\hline $2-4$ & 51.9 & 51.9 & 53.3 & \\
\hline \multicolumn{5}{|l|}{ Self-reported history of past spirometry } \\
\hline Yes & 79.7 & 83.7 & 84.0 & 0.012 \\
\hline No & 20.3 & 16.3 & 16.0 & \\
\hline \multicolumn{5}{|l|}{ Number of comorbidities } \\
\hline $0-1$ & 61.5 & 61.4 & 66.6 & 0.031 \\
\hline$\geq 2$ & 38.5 & 38.6 & 33.4 & \\
\hline \multicolumn{5}{|l|}{ Number of COPD medications } \\
\hline $0-1$ & 52.6 & 49.4 & 48.5 & 0.061 \\
\hline$\geq 2$ & 47.4 & 50.6 & 51.5 & \\
\hline \multicolumn{5}{|l|}{ Emergency department visits for COPD in past } \\
\hline \multicolumn{5}{|l|}{12 months } \\
\hline None & 68.5 & 69.3 & 76.6 & $<0.001$ \\
\hline$\geq 1$ & 31.5 & 30.7 & 23.4 & \\
\hline \multicolumn{5}{|l|}{ Hospital admissions for COPD in past 12 months } \\
\hline None & 80.9 & 78.6 & 83.1 & 0.314 \\
\hline$\geq 1$ & 19.1 & 21.4 & 16.9 & \\
\hline \multicolumn{5}{|l|}{ Satisfaction with doctors management of COPD } \\
\hline Very satisfied & 44.7 & 54.9 & 63.8 & $<0.000 \mathrm{I}^{\#}$ \\
\hline \multicolumn{5}{|l|}{ Involved in decisions about treatment } \\
\hline Strongly agree & 43.2 & 49.2 & 60.4 & $<0.000 I^{\#}$ \\
\hline \multicolumn{5}{|l|}{ Treatment plan or goals set up } \\
\hline Strongly agree & 38.5 & 42.3 & 53.0 & $<0.000 \mathrm{I}^{\#}$ \\
\hline \multicolumn{5}{|l|}{ Education level } \\
\hline Elementary school & 54.8 & 61.0 & 57.7 & 0.134 \\
\hline Higher education/technical college or further education & 45.2 & 39.0 & 42.3 & \\
\hline
\end{tabular}

Notes: ^Presented as column weighted percent unless otherwise stated; *tested across MMAS-8 levels using weighted chi-square test, proportional odds model or weighted ANOVA; \#P-value derived across all response categories.

Abbreviations: SD, standard deviation; CAT, COPD Assessment Test; mMRC, modified Medical Research Council.

chronic debilitating diseases ${ }^{20-23}$ however, we identified only one other study reporting data on patient engagement in COPD..$^{24}$ This study of 205 COPD patients, which focused on retraining of health behaviors, showed a mean PAM-13 score at baseline (59.0), which is lower than that reported in our study (66.75).
When considering medication adherence, it has been reported that in developed countries, $50 \%$ of patients with chronic disease do not take their medications as prescribed. ${ }^{25}$ This level of nonadherence has also been reported in COPD patients receiving home nebulizer therapy. ${ }^{26,27}$ George et al ${ }^{28}$ used a self-administered questionnaire (the medication 


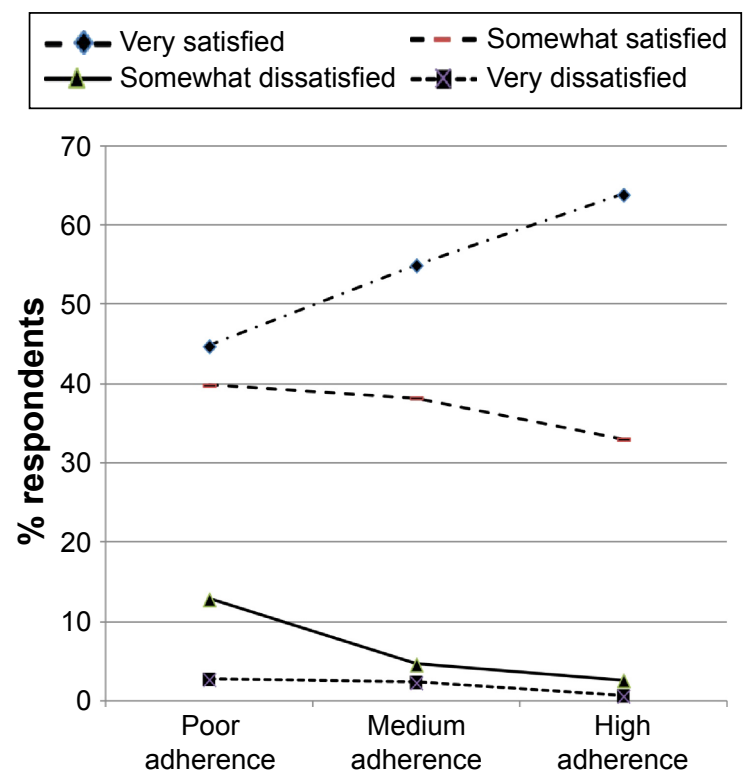

Figure 2 Satisfaction of patients with their treating physicians stratified by Morisky Medication Adherence Scale (MMAS)-8.

Note: Respondent \% based on MMAS-8 level, that is, sum of proportions within each MMAS-8 level add up to $100 \%$.

Table 4 Factors associated with low adherence (Morisky Medication Adherence Scale score $<6$ ) among respondents with COPD

\begin{tabular}{|c|c|}
\hline Effect & $\begin{array}{l}\text { Odds ratio point } \\
\text { estimate }(95 \% \text { Wald } C L)\end{array}$ \\
\hline \multicolumn{2}{|l|}{ Age (years) } \\
\hline $40-59$ & $1.88(1.55-2.29)^{*}$ \\
\hline Age $\geq 60$ & Ref \\
\hline \multicolumn{2}{|l|}{ Sex } \\
\hline Female & $1.08(0.88-1.32)$ \\
\hline Male & Ref \\
\hline \multicolumn{2}{|l|}{ Smoking status } \\
\hline Former smoker & $1.04(0.82-1.32)$ \\
\hline Current & $1.65(1.27-2.14)^{*}$ \\
\hline Never & Ref \\
\hline \multicolumn{2}{|l|}{ Emergency department visits } \\
\hline \multicolumn{2}{|l|}{ for COPD, prior year } \\
\hline None & Ref \\
\hline$\geq 1$ & I.0I (0.80-I.27) \\
\hline \multicolumn{2}{|l|}{ Satisfaction with doctors } \\
\hline Very satisfied & Ref \\
\hline Somewhat satisfied & $1.25(1.00-1.56)$ \\
\hline Somewhat/very dissatisfied & $2.7 \mathrm{I}(1.91-3.85)^{*}$ \\
\hline \multicolumn{2}{|l|}{ CAT score } \\
\hline Per 2-unit increment & I.0I (0.98-I.03) \\
\hline \multicolumn{2}{|c|}{ Self-reported history of past spirometry } \\
\hline Yes & $0.91(0.69-1.18)$ \\
\hline No & Ref \\
\hline \multicolumn{2}{|l|}{ Number of comorbidities } \\
\hline $0-1$ & Ref \\
\hline$\geq 2$ & $1.25(1.01-1.55)^{*}$ \\
\hline
\end{tabular}

Notes: The multivariate model was further adjusted for individual country. *This variable remained statistically significant in the multivariate model $(95 \% \mathrm{CL}$ does not include I, $P<0.05$ )

Abbreviations: CAT, COPD Assessment Test; CL, confidence limits. adherence report scale) to assess adherence in COPD patients identified through respiratory support groups and from a pulmonary rehabilitation database, and also reported a higher prevalence of nonadherence $(63 \%)$ than that reported in the current survey. Another recently published study evaluating the adherence of COPD patients in a clinical setting using the MMAS-4 reported poor adherence among $29.5 \%$ of COPD patients, an estimate comparable with our survey. ${ }^{12}$ These examples illustrate the reported variability in the prevalence of adherence to treatment in COPD when assessed using a range of methods or instruments in differing populations.

Both of these traits, poor patient engagement and adherence, were related to low levels of satisfaction with COPD management by health care professionals, poorer COPDspecific health status (CAT score), and potentially either more severe or less well-controlled disease as indicated by more frequent emergency department visits. In a multivariate model environment, satisfaction with their doctors' management of COPD appeared as the major factor impacting both engagement and adherence. Patients satisfied with their doctors as well as those feeling more actively involved in decisions about their treatment reported higher engagement and adherence scores. Consistent with this, George et $\mathrm{al}^{28}$ reported that the amount of time doctors spent with patients was a key determinant of medication adherence. Several studies have highlighted that establishing a good doctor-patient relationship is necessary for fostering patient engagement in the consultation. ${ }^{29,30}$

Additional factors independently associated with low engagement were poorer COPD-specific health status (CAT) and the level of reported breathlessness as measured with mMRC. Breathlessness has been described as the most troublesome symptom by patients with COPD and is a leading cause of disability. ${ }^{31}$ While poor engagement was associated with poor COPD-specific health status and COPD symptoms (breathlessness) in multivariate modeling, these relationships were not maintained with the medication adherence outcome in multivariate modeling. Similarly, the relationship between prior health care utilization for COPD exacerbations (hospital/emergency department visits), although related to both low engagement and poor adherence on univariate testing, was attenuated for both in the multivariate model. Several factors could have contributed to such observations: patients with high engagement level could potentially represent a mixed population of patients from the extremes of the disease, that is, those with a low disease severity as well as those with severe dyspnea and poor health status. This hypothesis will be addressed in a further analysis. The potential lack of temporality of self-reported prior emergency department visits and 
hospitalizations for COPD and PAM-13 and MMAS-8 assessments limits the ability to study any mutual relationships. Further, health care utilization patterns significantly differ across regions and the three geographical areas of Asia, South America, and US/Europe represented in this survey may have introduced heterogeneity. We also found a relationship between BMI (underweight, overweight, and obese) and low patient engagement. Such a relationship was described by Fowles et al in a cohort of US employees. ${ }^{32}$ In their analysis, lower BMI predicted higher activation. We have treated BMI as a nonlinear variable, based on our a priori knowledge of an association of BMI with patient reported outcomes in COPD; ${ }^{33}$ hence, we have shown that being underweight, often associated with poorer outcomes in COPD, is also related to poorer activation.

Other factors that were independent predictors of poor adherence were current smokers, younger age ( $<65$ years), and presence of $\geq 2$ comorbidities. A cross-sectional survey of employees from a large US company, using a medical and pharmacy claims database, also showed for a range of chronic conditions that smokers were significantly less adherent to medications than nonsmokers. ${ }^{34}$ Better adherence in older patients with chronic diseases, including COPD, has been shown in other studies. ${ }^{11,35}$ Further, others have reported on an association between increased number of comorbidities and poorer adherence. ${ }^{11}$ This is an important point for treating physicians. As the numbers of comorbidities increase, so too typically do the number of medications prescribed to a patient. Our data suggest these represent "at risk patients" and in particular those who are poorly engaged may be even more likely to be nonadherent to complex and frequently expensive drug regimens.

Improved patient engagement may be expected to translate into better control of disease and result in lower disease burden. However, the Continuing to Confront COPD International Patient Survey provides cross-sectional data so we cannot infer the impact of these findings on long-term outcomes in COPD patients. In the area of management of diabetes, patient engagement interventions resulted in modest improvements in intermediate outcomes, such as hemoglobin A1c, but the evidence supporting clinically important outcomes was considered as weak. ${ }^{36}$ One study in COPD assessed the impact of repeated training sessions on patient engagement, disease symptom burden, and health-related quality of life. ${ }^{24}$ Although the intervention significantly improved the level of engagement, disease burden and health-related quality of life remained unchanged, highlighting the need for additional research. For patients, this may involve managing their COPD more effectively or could also be a case of better management of their comorbidities, which may also result in better overall health and outcomes. ${ }^{37}$ However, at the highest level of engagement, patients may still struggle to maintain healthy behaviors but report having the skills and confidence to manage their health more proactively, which may in turn reduce the effect of any intervention. ${ }^{19}$

A limitation of our results and their interpretation is that the survey sampling method recruited patients from the general population which may have resulted in a patient group reporting milder disease than that usually observed in a health care setting. A quarter of patients had not taken any medications for COPD in the 2 weeks prior to the survey which may reflect a milder population but which could also have been related to cultural variation in access to medication. In addition, selection bias is another potential limitation of this type of population-based survey. While a large international span across three continents increased the representativeness of the results, it is also likely to have introduced heterogeneity with respect to cultural aspects of expected patient and health care provider roles, and differences in access to health care. In addition, we assessed disease burden using only a limited number of measures, mainly patient self-reported outcomes. Approximately $80 \%$ of the population self-reported that they had undergone a past spirometry test but confirmation of spirometric diagnosis of COPD by a review of patient records was not within the scope of this survey. Although a third of our patient population reported that they had never smoked, which is traditionally atypical of a diagnosed COPD population, a review by Zeng et $\mathrm{al}^{38}$ also reported the high burden of COPD in nonsmokers, highlighting the variation in its prevalence across regions. The frequency of nonsmokers in this study is still higher than in any study in the review by Zeng et al in Western countries (around 23\%), but relatively comparable to the US National Health and Nutrition Examination Surveys ( $32 \%$ for patients with spirometry results suggestive of airway obstruction). ${ }^{38,39}$ Finally, despite collecting extensive data on patient characteristics, we cannot rule out the fact that unmeasured and important confounding factors may have accrued and impacted our findings.

\section{Conclusion}

About a third of patients with COPD sampled from the general population do not feel engaged or involved with their COPD management and often report nonadherence with treatment. COPD patients reporting disengagement and 
lack of adherence to COPD medications also report greater symptom burden and less satisfaction with their COPD management. Further research is needed to identify how best to help these patients and whether psychosocial, cultural, and/ or biological factors are driving these associations.

\section{Acknowledgments}

The survey was conducted by Abt SRBI, a global survey research firm. The authors would like to acknowledge editorial support in the form of draft outline manuscript development, assembling tables, collating author comments, and copyediting which was provided by Kate Hollingworth of Continuous Improvement Ltd. This editorial support was funded by GSK. This study was funded by GSK.

\section{Author contributions}

All authors meet the International Committee for Medical Journal Editors criteria for authorship. HM, the lead author and guarantor, contributed to the concept and design of the survey, developing the analysis plan for this paper, and took a lead role in interpreting the results and writing the manuscript. SHL, KJD, JM, and MT contributed to the concept and design of the survey, interpreting the results, and writing the manuscript. ZA, MI, DMM, AMM, TvdM, $\mathrm{Y}-\mathrm{MO}$, and $\mathrm{MKH}$ served on the Scientific Advisory Committee for the Continuing to Confront COPD Survey and contributed to the concept and design of the survey, interpreting the results and writing the manuscript. JM provided statistical analysis support. All authors reviewed and approved the final manuscript.

\section{Disclosure}

HM, SHL, KJD, and MT are employees of GSK and hold GSK shares. At the time of writing, JM was a contract statistician for GSK. ZA, MI, DMM, AMM, TvdM, Y-MO, and MKH served on the Scientific Advisory Committee for the Continuing to Confront COPD Survey and were paid for advisory services. Scientific Advisory Committee members were not paid for authorship service. There are no other conflicts of interest to declare.

\section{References}

1. Snoek FJ. Breaking the barriers to optimal glycaemic control - what physicians need to know from patients' perspectives. Int J Clin Prac Suppl. 2002;129:80-84.

2. Hibbard JH, Mahoney ER, Stockard J, Tusler M. Development and testing of a short form of the patient activation measure. Health Serv Res. 2005;40(6):1918-1930.

3. Hibbard JH, Mahoney ER, Stock R, Tusler M. Self-management and health care utilization: do increases in patient activation result in improved selfmanagement behaviours? Health Serv Res. 2007;42(4):1443-1463.
4. Rask KJ, Ziemer DC, Kohler SA, Hawley JN, Arinde FJ, Barnes C. Patient activation is associated with healthy behaviours and ease in managing diabetes in an indigenous population. Diabetes Educ. 2009; 35:622-630.

5. Adams SG, Smith PK, Allan PF, Anzueto A, Pugh JA, Cornell JE. Systematic review of the chronic care model in chronic obstructive pulmonary disease prevention and management. Arch Intern Med. 2007; 167:551-561.

6. Fromer L. Implementing chronic care for COPD: planned visits, care coordination, and patient empowerment for improved outcomes. Int J Chron Obstruct Pulmon Dis. 2011:6:605-614.

7. Vestbo J, Anderson JA, Calverley PM, et al. Adherence to inhaled therapy, mortality and hospital admission in COPD. Thorax. 2009;64: 939-943.

8. Simoni-Wastila L, Wei YJ, Qian J, et al. Association of chronic obstructive pulmonary disease maintenance medication adherence with all-cause hospitalization and spending in a Medicare population. Am J Geriatr Pharmacother. 2012;10:201-210.

9. Cramer JA, Bradley-Kennedy C, Scalera A. Treatment persistence and compliance with medications for chronic obstructive pulmonary disease. Can Respir J. 2007;14:25-29.

10. Krigsman K, Nilsson JL, Ring L. Refill adherence for patients with asthma and COPD: comparison of a pharmacy record database with manually collected repeat prescriptions. Pharmacoepidemiol Drug Saf. 2007; $16: 441-448$

11. Bourbeau J, Bartlett SJ. Patient adherence in COPD. Thorax. 2008; 63:831-838.

12. Khdour MR, Hawwa AF, Kidney JC, Smyth BM, McElnay JC. Potential risk factors for medication non-adherence in patients with chronic obstructive pulmonary disease (COPD). Eur J Clin Pharmacol. 2012; 68:1365-1373.

13. Agh T, Inotai A, Meszaros A. Factors associated with medication adherence in patients with chronic obstructive pulmonary disease. Respiration. 2011;82:328-334.

14. Landis SH, Muellerova H, Mannino DM, et al. Continuing to Confront COPD International Patient Survey: methods, COPD prevalence, and disease burden in 2012-2013. Int J Chron Obstruct Pulmon Dis. 2014;9: 597-611.

15. Bestall JC, Paul EA, Garrod R, Garnham R, Jones PW, Wedzicha JA. Usefulness of the Medical Research Council (MRC) dyspnoea scale as a measure of disability in patients with chronic obstructive pulmonary disease. Thorax. 1999;54:581-586.

16. Jones PW, Harding G, Berry P, et al. Development and first validation of the COPD Assessment Test. Eur Respir J. 2009;34:648-654.

17. Jones PW, Harding G, Wiklund I, et al. Tests of the responsiveness of the Chronic Obstructive Pulmonary Disease (COPD) assessment Test ${ }^{\mathrm{TM}}$ (CAT) following acute exacerbations and pulmonary rehabilitation. Chest. 2012:142:134-140.

18. Morisky DE, Ang A, Krousel-Wood M, Ward HJ. Predictive validity of a medication adherence measure in an outpatient setting. $J$ Clin Hypertens. 2008;10:348-354.

19. Hibbard JH, Cunningham PJ. How engaged are consumers in their health and health care, and why does it matter? Res Brief. 2008; $8: 1-9$.

20. Hendriks M, Rademakers J. Relationships between patient activation, disease-specific knowledge and health outcomes among people with diabetes; a survey study. BMC Health Serv Res. 2014;14:393.

21. Skolasky R, Green AF, Scharfstein D, Boult C, Reider L, Wegener ST Psychometric properties of the patient activation measure among multimorbid older adults. Health Serv Res. 2011;46:2.

22. Kukla M, Salyers MP, Lysaker PH. Levels of patient activation among adults with schizophrenia: associations with hope, symptoms, medication adherence, and recovery attitudes. J Nerv Ment Dis. 2013; 201:339-344.

23. Sacks RM, Greene J, Hibbard JH, Overton V. How well do patient activation scores predict depression outcomes one year later? $J$ Affect Disord. 2014;169:1-6. 
24. Turner A, Anderson J, Wallace L, Kennedy-Williams P. Evaluation of a self-management programme for patients with chronic obstructive pulmonary disease. Chron Respir Dis. 2014;11:163-172.

25. World Health Organisation. Adherence to Long-Term Therapies: Evidence for Action. Available from: http://www.who.int/chp/knowledge/ publications/adherence_report/en/. Accessed September 10, 2015.

26. Corden ZM, Bosley CM, Rees PJ, et al. Home nebulized therapy for patients with COPD: patient compliance with treatment and its relation to quality of life. Chest. 1997;112:1278-1282.

27. Turner J, Wright E, Mendella L, Anthonisen N. Predictors of patient adherence to long-term home nebulizer therapy for COPD. The IPPB Study Group. Intermittent Positive Pressure Breathing. Chest. 1995;108: 394-400.

28. George J, Kong DC, Thoman R, Stewart K. Factors associated with medication nonadherence in patients with COPD. Chest. 2005;128: 3198-3204

29. Bastiaens H, Van Royen P, Pavlic DR, Raposo V, Baker R. Older people's preferences for involvement in their own care: a qualitative study in primary health care in 11 European countries. Patient Educ Couns. 2007;68:33-42.

30. Edwards A1, Elwyn G, Smith C, Williams S, Thornton H. Consumers' views of quality in the consultation and their relevance to 'shared decision-making' approaches. Health Expect. 2001;4:151-161.

31. Kessler R, Partridge MR, Miravitlles M, et al. Symptom variability in patients with severe COPD: a pan-European cross-sectional study. Eur Respir J. 2011;37(2):264-272.
32. Fowles JB1, Terry P, Xi M, Hibbard J, Bloom CT, Harvey L. Measuring self-management of patients' and employees' health: further validation of the Patient Activation Measure (PAM) based on its relation to employee characteristics. Patient Educ Couns. 2009;77(1):116-122.

33. Müllerová H, Lu C, Li H, Tabberer M. Prevalence and burden of breathlessness in patients with chronic obstructive pulmonary disease managed in primary care. PLoS One. 2014;9(1):e85540.

34. Sherman BW, Lynch WD. The association of smoking with medical treatment adherence in the workforce of a large employer. Patient Prefer Adherence. 2014;8:477-486.

35. Rolnic SJ, Pawloski PA, Hedblom BD, Asche SE, Bruzek RJ. Patient characteristics associated with medication adherence. Clin Med Res. 2013;11:54-65.

36. Bolen SD, Chandar A, Falck-Ytter C, et al. Effectiveness and safety of patient activation interventions for adults with type 2 diabetes: systematic review, meta-analysis, and meta-regression. J Gen Intern Med. 2014;29:1166-1176.

37. Barnes PJ, Celli BR. Systemic manifestations and comorbidities of COPD. Eur Respir J. 2009;33:1165-1185.

38. Zeng G, Sun B, Zhong N. Non-smoking-related chronic obstructive pulmonary disease: a neglected entity? Respirology. 2012;17:908-912.

39. Ford ES, Mannino DM, Wheaton AG, Giles WH, Presley-Cantrell L, Croft JB. Trends in the prevalence of obstructive and restrictive lung function among adults in the United States: findings from the National Health and Nutrition Examination surveys from 1988-1994 to 2007-2010. Chest. 2013;143:1395-1406.
International Journal of COPD

\section{Publish your work in this journal}

The International Journal of COPD is an international, peer-reviewed journal of therapeutics and pharmacology focusing on concise rapid reporting of clinical studies and reviews in COPD. Special focus is given to the pathophysiological processes underlying the disease, intervention programs, patient focused education, and self management protocols.

\section{Dovepress}

This journal is indexed on PubMed Central, MedLine and CAS. The manuscript management system is completely online and includes a very quick and fair peer-review system, which is all easy to use. Visit http://www.dovepress.com/testimonials.php to read real quotes from published authors. 